Research Paper

\title{
Glycogen Synthase Kinase $3 \beta$ Influences Injury Following Cerebral Ischemia/Reperfusion in Rats
}

\author{
Yixin Li1 ${ }^{1,2,3^{*}}$, Jin Zhu ${ }^{1,2,3^{*}}$, Yuanling Liu ${ }^{1,2,3}$, Xi Chen ${ }^{1,2,3}$, Shipeng Lei ${ }^{4}$, Lingyu Li1 ${ }^{1,2,3}$, Beibei Jiang1,2,3, Li \\ $\operatorname{Tan}^{1,2,3}$, Shanshan $\mathrm{Yu}^{1,2,3}$, Yong Zhao ${ }^{1,2,3}$ \\ 1. Department of Pathology, Chongqing Medical University, Chongqing, People's Republic of China; \\ 2. Institute of Neuroscience, Chongqing Medical University, Chongqing, People's Republic of China; \\ 3. Key Laboratory of Neurobiology, Chongqing Medical University, 400016 Chongqing, People's Republic of China; \\ 4. Department of Respiratory Medicine, Jiangjin Center Hospital, 402260, Chongqing, People's Republic of China. \\ * Yixin Li and Jin Zhu contributed equally to this study. \\ $\triangle$ Corresponding authors: Shanshan Yu Or Yong Zhao. Department of Pathology, Chongqing Medical University, Yixueyuan Road 1, 400016 Chongqing, \\ People's Republic of China. Fax: 86-23-68485868 Telephone: 86-23-68485789 E-mail address: yushanshan2014@cqmu.edu.cn (Shanshan Yu) Or \\ zhaoyong668@cqmu.edu.cn (Yong Zhao).
}

(C) Ivyspring International Publisher. Reproduction is permitted for personal, noncommercial use, provided that the article is in whole, unmodified, and properly cited. See http://ivyspring.com/terms for terms and conditions.

Received: 2015.09.20; Accepted: 2015.12.29; Published: 2016.02.29

\begin{abstract}
Abnormal activation of GSK-3 $\beta$ is associated with psychiatric and neurodegenerative disorders. However, no study has examined the effect of GSK-3 $\beta$ on cerebral ischemia/reperfusion injury. We used oxygen-glucose deprivation/reoxygenation (OGD/R) and middle cerebral artery occlusion (MCAO) as models of ischemia/reperfusion in rats in vitro and in vivo. Our study showed that knockdown of GSK-3 $\beta$ with a GSK-3 $\beta$ siRNA virus improved injury and increased viability of neurons subjected to OGD/R. Levels of total Nrf2, nuclear Nrf2, and Nrf2 downstream proteins sulfiredoxin (Srxl) and thioredoxin (Trxl) increased after transfection with the GSK-3 $\beta$ siRNA virus. GSK-3 $\beta$ siRNA increased SOD activity and decreased MDA levels. Overexpression of GSK-3 $\beta$ with a pcDNA-GSK-3 $\beta$ virus showed opposite results. We also demonstrated that intracerebroventricular injection of GSK-3 $\beta$ siRNA in rats ameliorated neurological deficits, reduced brain infarct volume and water content, and reduced damage to cerebral cortical neurons after MCAO. Changes in total Nrf2, nuclear Nrf2, Srxl, Trx1, SOD, and MDA were similar to those observed in vitro. Our results show for the first time that GSK-3 $\beta$ can influence cerebral ischemia/reperfusion injury. The effects may be due to regulating the Nrf2/ARE pathway and decreasing oxidative stress. These results suggest a potential new drug target for clinical treatment of stroke.
\end{abstract}

Key words: glycogen synthase kinase-3 $\beta$; cerebral ischemia/reperfusion injury; NF-E2-related factor 2.

\section{Introduction}

Stroke is a serious concern because it poses a serious threat to the life and health of humans $[1,2]$. The brain is the most sensitive organ to hypoxia, and cerebral ischemia-reperfusion can cause severe damage to brain functions. Many studies have demonstrated that oxidative stress plays a key role in injury of cortical neurons after brain ischemia. Reactive oxygen species (ROS) that are produced by oxidative stress damage physiological function of neurons directly or indirectly. However, exogenous medications exhibit undesirable therapeutic effects. Activation of the endogenous antioxidant system is crucial for the survival of neurons after ischemia/reperfusion.

A previous study has demonstrated that GSK-3 $\beta$ participates in acute hepatic damage induced by oxidative stress [3]. We hypothesize that GSK-3 $\beta$ may influence cerebral ischemia/reperfusion injury. GSK-3 $\beta$ is a serine/threonine kinase that is evolutionarily conserved. It is widespread in mammalian eukaryotic cells and has effects on many signaling 
proteins and transcription factors. Abnormal activation of GSK-3 $\beta$ is associated with psychiatric and neurodegenerative disorders [4]. In addition, it has been demonstrated that both preconditioning $[5,6]$ and postconditioning [7], which involve administration of GSK-3 $\beta$ inhibitors before ischemia (preconditioning) or just before reperfusion (postconditioning), decreased infarct size in the myocardium $[8,9]$.

Studies also confirm that GSK-3 $\beta$ participates in regulation of subcellular translocation of $\mathrm{Nrf} 2$ to the nucleus in various cells [10]. Nrf2 (NF - E2 - related factor 2), an important member of the endogenous antioxidant system, is a key factor in the oxidative stress response [11]. It regulates expression of phase II detoxifying enzymes and antioxidants such as sulfiredoxin (Srx1) [12] and thioredoxin (Trx1) [13] by interacting with the antioxidant response element (ARE). This induction involves mechanisms that are significant for the protection of cells against neoplastic diseases, as well as oxidative and electrophilic stress [14]. Nrf2 is localized to the cytoplasm in most normal cells. In response to oxidative stress or various other stresses, the expression of Nrf2 increases and the protein accumulates in the nucleus. In the nucleus, Nrf2 binds to ARE and activates expression of antioxidants that antagonize injury induced by oxidative stress [15]. Nrf2 is critical for the protection of neurons from oxidative injury induced by ische$\mathrm{mia} /$ reperfusion as described in our previous study [16]. Thus, activity of Nrf2 must be stringently controlled. Previous studies have suggested that Nrf2 is primarily regulated by Keap1. However, current studies indicate that other regulatory factors such as GSK-3 $\beta$ participate in regulation of Nrf2 independently of Keap1.

Research has shown that GSK-3 $\beta$ interference increased accumulation of Nrf2 in the nucleus when human hepatoma (HepG2) cells were transfected with GSK-3 $\beta$ siRNA [17]. In addition, GSK-3 $\beta$ can expel Nrf2 from the nucleus in human embryo kidney 293T (HEK293T) cells [18]. A previous study also showed that GSK-3 $\beta$ downregulated Nrf2 when neuronal cells were exposed to $\mathrm{H}_{2} \mathrm{O}_{2}$ [19]. However, the effects of GSK-3 $\beta$ on damage due to cerebral ische$\mathrm{mia} /$ reperfusion and on neurons subjected to OGD/R have not been explored.

Based on previous research, we hypothesized that GSK-3 $\beta$, as a critical regulatory factor of Nrf2, may influence injury induced by cerebral ische$\mathrm{mia} /$ reperfusion and OGD/R. To verify our hypotheses and explore a new target for treatment of stroke, we conducted MCAO in vivo and OGD/R in vitro in the cerebral cortex of rats and cultured cortical neurons, respectively.

\section{Materials and Methods}

\section{Animals and Agents}

Adult male Sprague-Dawley rats $(250-300$ g) were used for the in vivo study. Newborn Sprague-Dawley rats (less than $24 \mathrm{~h}$ old) were used in the in vitro study. All experiments were approved by the Institutional Animal Care and Use Committee of the Chongqing Medical University.

GSK-3 $\beta$ lentivirus was purchased from Neuronbiotech (Shanghai, China). Leptomycin B and a lactate dehydrogenase (LDH) kit were purchased from Beyotime Biotechnology (Shanghai, China). Curcumin was used as a positive control and obtained from Sigma-Aldrich (St Louis. MO. USA). Nrf2 antibody was obtained from ImmunoWay (Newark, DE, USA). Neurobasal Medium, supplement B27, and glucose-free Dulbecco's Modified Eagle's Medium (DMEM) were purchased form Gibco BRL (Grand Island, NY, USA). MTS and 2,3,5-triphenyl tetrazolium chloride (TTC) were obtained from Sigma-Aldrich. General reagents were purchased from Sigma-Aldrich and other commercial suppliers.

\section{Primary Culture of Rat Cortical Neurons and OGD/R}

Cortical neurons were prepared from newborn Sprague-Dawley rat brain tissue as previously described [20, 21]. The cells were cultured in medium containing 97\% Neurobasal Medium, 2\% B27, 1\% penicillin and streptomycin. Neurons were cultured for $6 \mathrm{~d}$ in vitro. To conduct OGD/R, the medium was replaced with a glucose-free DMEM solution and cultures were transferred to a tri-gas incubator (Forma model 3131, Thermo Scientific, Marietta, OH, USA) with an atmosphere of $94 \% \mathrm{~N}_{2}, 1 \% \mathrm{O}_{2}$ and $5 \% \mathrm{CO}_{2}$ for $1.5 \mathrm{~h}$ at $37{ }^{\circ} \mathrm{C}$. After $1.5 \mathrm{~h}$ of OGD, the medium was replaced with 97\% Neurobasal Medium. 2\% B27, 1\% penicillin and streptomycin and cultures were returned to the normal incubator for $1 \mathrm{~h}$ or $24 \mathrm{~h}$ of reoxygenation. The purity of the cultured cells was determined by NeuN and GFAP staining to ensure that the cells were at least $90 \%$ neurons.

We divided neurons without $\mathrm{OGD} / \mathrm{R}$ into 5 groups: Control group, vector group, GSK-3 $\beta$ siRNA group, GSK-3 $\beta$ overexpression group, and curcumin-treated group. We divided neurons with OGD/R into 7 groups: OGD/R group, vector + OGD/R group, GSK-3 $\beta$ siRNA + OGD/R group, GSK-3 $\beta$ overexpression + OGD/R group, curcumin-treated + OGD/R group, leptomycin B-treated + OGD/R group, GSK-3 $\beta$ overexpression + leptomycin B-treated + OGD/R group. Neurons were treated with leptomycin $\mathrm{B}(10 \mu \mathrm{M})$ for $6 \mathrm{~h}$ or curcumin $(5 \mu \mathrm{M})$ for $24 \mathrm{~h}$ before OGD [16]. 


\section{GSK-3 $\beta$ Interference and Overexpression in Neurons}

GSK-3 $\beta$ siRNA lentiviral vectors were constructed by Neuronbiotech. The rat GSK-3 $\beta$ gene sequence (accession number: NM 032080.1) is available in the NCBI database.

GSK-3 $\beta$ siRNA (GCTAGATCACTGTAACATA GT) was designed and synthesized as follows: 5'CCGGGCTAGATCACTGTAACATAGTCTCGAGAC TATGTTACAGTGATCTAGCTTTTTTG-3' (sense); 5'-AATTCAAAAAAGCTAGATCACTGTAACATAG TCTCG-AGACTATGTTACAGTGATCTAGC-3' (antisense). These oligonucleotides were annealed and inserted downstream of the U6 promoter on the lentiviral vector pLKD.UBC.GFP (Neuronbiotech). Lentivirus was harvested after transfection of HEK293T cells.

The GSK-3 $\beta$ insert was isolated by PCR amplification from pcDNA-GSK-3 $\beta$ using 2 primers. PCR products were sequenced and confirmed (Invitrogen, Carlsbad, CA, USA) to contain the entire GSK-3 $\beta$ coding sequence. The GSK-3 $\beta$ overexpression lentivirus was packaged using the pLOV-UbiC-EGFP vector and then $293 \mathrm{~T}$ cells were transfected with the expression vectors. The GSK-3 $\beta$ overexpression lentivirus was harvested, filtered, and concentrated.

After $3 \mathrm{~d}$ in culture, neurons were transfected with lentivirus for $72 \mathrm{~h}$. The multiplicity of infection (MOI) of the GSK-3 $\beta$ siRNA lentivirus was 10 , and the MOI of the GSK-3 $\beta$ overexpression lentivirus was 20. Transfection efficiency was assessed under a fluorescence microscope. Sustained GSK-3 $\beta$ overexpression and interference were confirmed by qRT-PCR and western blot analyses (data not shown).

\section{Cell Viability and Injury Assay}

To assess the viability of cells, we used colorimetric 3-(4,5-dimethylthiazol-2yl)-5-(3-carboxymethoxyphenyl)-(4-sulfophenyl)-2H-tetrazolium (MTS). Neurons were cultured in 96-well plates. After $3 \mathrm{~d}$, neurons were treated with GSK-3 $\beta$ overexpression lentivirus, GSK-3 $\beta$ siRNA lentivirus, or vector lentivirus for $72 \mathrm{~h}$ before being subjected to OGD/R. After $24 \mathrm{~h}$ of reoxygenation, the medium was collected for $\mathrm{LDH}$ detection and cells were subjected to the MTS assay. MTS solution was added to fresh medium (1 $\mathrm{mg} / \mathrm{mL}$ ). After neurons were cultured with the fresh medium for $2 \mathrm{~h}$, cells were measured on a microplate reader at a wavelength of $570 \mathrm{~nm}$ (Bio-Rad, Foster City, CA, USA).

An LDH release assay was used to assess cytotoxicity of neurons after OGD/R. We used a commercially available kit to measure LDH activity (Nanjing Jiancheng Bioengineering Institute, Nanjing,
China). Absorbance was detected at $440 \mathrm{~nm}$ on microplate reader.

\section{MCAO Model}

The MCAO model was established in adult male Sprague-Dawley rats (250-300 g) as described previously [22, 23]. Briefly, rats were anesthetized with $3.5 \%$ chloral hydrate $(350 \mathrm{mg} / \mathrm{kg}$, intraperitoneally). A plug (Shadong Biotechnology Company, Beijing, China) was inserted into the left internal carotid artery through the external carotid artery and advanced 18-20 mm to block middle cerebral artery. After $1 \mathrm{~h}$ of ischemia, the plug was removed from middle cerebral artery to restore blood flow. After $6 \mathrm{~h}$ of reperfusion, tissue from the left cerebral cortex was subjected to western blot analysis. In addition, after $24 \mathrm{~h}$ of reperfusion, the brains were subjected to TTC, hematoxylin-eosin (HE), and Nissl staining. In the sham group, the plug was not inserted into the middle cerebral artery and remained in the external carotid artery for $1 \mathrm{~h}$.

\section{GSK-3 $\beta$ Interference in Rats}

The GSK-3 $\beta$ siRNA oligonucleotide (sense primer 5-GGAGAGCCCAAUGUUUCAUTT-3) and (antisense primer 5-AUGAAACAUUGGGCUCUCCTT3) was designed and synthesized by GenePharma Corporation (Shanghai, China). As a control, a scramble sequence (sense primer 5-GCGCCAGUG GUACUUAAUATT-3) and (antisense primer 5-UAUUAAGUACCACUGGCGCTT-3) without any target sequence was synthesized. GSK-3 $\beta$ siRNAs were injected into the left lateral cerebral ventricle 48 $\mathrm{h}$ before MCAO. A fluorescence microscope was used to detect transfection efficiency. The siRNA injection method was described in detail in our previous study [24]. Knockdown efficiency of GSK-3 $\beta$ siRNA was confirmed by qRT-PCR and western blot analysis (data not shown).

Adult male Sprague-Dawley rats were divided randomly into the following 8 groups: control group, scramble group, GSK-3 $\beta$ siRNA group, curcumin-treated group, sham-operated group, MCAO group, scramble + MCAO group, GSK-3 $\beta$ siRNA + MCAO group, and curcumin $(300 \mathrm{mg} / \mathrm{kg}$, intraperitoneally) + MCAO group [16]

\section{HE and Nissl Staining}

HE and Nissl staining were conducted to assess morphological changes. Brains were fixed with $4 \%$ paraformaldehyde, followed by dehydration, dipping in wax, embedding, and sectioning. The sections were stained with HE or cresyl violet (Nissl staining). Morphological changes were assessed by a pathologist from the Pathological Diagnosis Center of Chongqing. 


\section{Measurement of Infarct Volume}

After $24 \mathrm{~h}$ of reperfusion, brain tissues were sliced into $2 \mathrm{~mm}$ thick sections and stained with $2 \%$ TTC at $37^{\circ} \mathrm{C}$ for $15 \mathrm{~min}$. Images were captured by a digital camera (Nikon E5100) and areas of infarct were calculated using Image J (version 1.37c; NIH, Bethesda, MD, USA). Percentage of infarct area was calculated using the following equation: (left infarct volume-left edema volume)/right volume $\times 100 \%$, where left edema volume $=$ left volume-right volume.

\section{Measurement of Neurologic Deficit Scores and Brain Water Content}

Neurologic deficit scores were assessed after $24 \mathrm{~h}$ of reperfusion using a modified scoring system that was developed by Longa et al. [25]. The scoring system was as follows: 0 , no neurological deficits; 1 , unable to extend right forepaw fully; 2 , circling to the right; 3 , falling to the right; 4 , loss of consciousness or dead.

Brain water content was measured by weighing the left and right brains. Rats were killed $24 \mathrm{~h}$ after reperfusion and brains were dissected into left and right hemispheres. They were then immediately weighed on an electronic balance (wet weight). After $72 \mathrm{~h}$ of drying in an oven at $70{ }^{\circ} \mathrm{C}$, the hemispheres were weighed again (dry weight). Brain water content of the hemispheres was calculated as follows: (wet weight - dry weight)/ wet weight $\times 100 \%$.

\section{SOD Activity and Measurement of MDA Lev- els}

To detect oxidative injury in neurons and rat brains, superoxide dismutase (SOD) activity and malondialdehvde (MDA) levels were measured in neuronal medium and experimental rat cortical tissue using a commercially available kit (Nanjing Jiancheng Bioengineering Institute, Nanjing, China) following the manufacturers' instructions as described previously [26]. Briefly, SOD activity was measured using the WST-1 method. Absorbance was determined at $450 \mathrm{~nm}$ by spectrometry. MDA level was measured by the thiobarbituric acid method. Absorbance was measured by spectrometry at $532 \mathrm{~nm}$. The concentration of brain tissue protein was detected using the Enhanced BCA Protein Assay Kit (Beyotime, Jiangsu, China).

\section{Western Blot Analysis}

Total protein and nuclear protein were extracted from cultured neurons and left cortical tissues at the indicated times. Total protein was extracted in lysis buffer that included phosphatase and protease inhibitors. Nuclear proteins were extracted using a commercially available kit (Beyotime Institute of Bio- technology, Haimen, Jiangshu, China). Membranes were incubated with the primary antibody overnight at $4{ }^{\circ} \mathrm{C}$. The primary antibody used was Nrf2 (1:500; ImmunoWay Biotechnology).

\section{Immunofluorescence Staining Analysis}

After deparaffinization, sections were incubated with $1 \%$ TritonX-100 for $15 \mathrm{~min}$ at $37{ }^{\circ} \mathrm{C}$ and then washed with PBS. Sections were subjected to high-pressure antigen retrieval for $30 \mathrm{~min}$. After they cooled, sections were incubated in normal goat serum at $37^{\circ} \mathrm{C}$ for $15 \mathrm{~min}$. Sections were then incubated in primary antibody (Nrf2, rabbit, 1:50; NeuN, mouse, 1:50) overnight at $4{ }^{\circ} \mathrm{C}$. The next day, sections were incubated in FITC-conjugated goat anti-rabbit IgG antibody (1:200) and anti-mouse IgG antibody (1:200) for $30 \mathrm{~min}$ at $37^{\circ} \mathrm{C}$. Neurons growing on glass slides were incubated with Nrf2 primary antibody (rabbit, 1:50) overnight at $4{ }^{\circ} \mathrm{C}$ and then stained with DAPI for $30 \mathrm{~min}$ at $37^{\circ} \mathrm{C}$. Sections and glass slides were imaged with a laser scanning confocal microscope (LSCM).

\section{Statistical Analysis}

All data are expressed as mean \pm SEM. Statistical comparisons were assessed with one-way analysis of variance (ANOVA) followed by Student's $t$ test and post hoc Fisher's test. The statistical software package SPSS 18.0 was used. A value of $\mathrm{P}<0.05$ was considered statistically significant.

\section{Results}

\section{Effects of GSK-3 $\beta$ on Injury and Survival of Cortical Neurons Subjected to OGD/R}

When neurons were subjected to OGD for $1.5 \mathrm{~h}$ followed by $24 \mathrm{~h}$ of reoxygenation, the release of $\mathrm{LDH}$ increased to $36.27 \pm 3.83 \%$ compared with the control group $(\mathrm{P}<0.01$, Fig. 1A). Compared with the OGD/R group, the increase in LDH was more significant in the vector $+\mathrm{OGD} / \mathrm{R}$ group. In the GSK-3 $\beta$ siRNA group, LDH release decreased to $27 \pm 3.94 \%$ compared with $61.43 \pm 5.69 \%$ in the vector + OGD group $(\mathrm{P}<0.01)$. In contrast, $\mathrm{LDH}$ release increased to 94.53 $\pm 3.17 \%(\mathrm{P}<0.01)$ in the GSK-3 $\beta$ overexpression group.

In the OGD/R group, MTS activity decreased to $47.67 \pm 3.18 \%$ compared with the control group $(\mathrm{P}<$ 0.01 , Fig. $1 B$ ). In the vector + OGD/R group, survival rate decreased to $37.67 \pm 4.33 \%$. In the GSK $-3 \beta$ siRNA + OGD/R group, survival rate increased to $58.67 \pm$ $4.37 \%$ compared with the vector + OGD $/ \mathrm{R}$ group $(\mathrm{P}<$ $0.01)$. In the GSK-3 $\beta$ overexpression group, the value decreased to $21.67 \pm 1.85 \%$ ( $\mathrm{P}<0.05)$.

These results suggest that GSK-3 $\beta$ interference reduced injury and improved viability of neurons 
subjected to OGD/R, whereas GSK-3 $\beta$ overexpression increased injury and reduced viability.

\section{Effects of GSK-3 $\beta$ on Neurological Deficits, Infarct Volume, and Brain Water Content}

Longa scores were used to evaluate neurological deficits caused by MCAO ( $\mathrm{n}=6$ per group). Compared with the scramble + MCAO group, neurological deficits and infarct volume decreased after rats were infected with GSK-3 $\beta$ siRNA 48 h before MCAO (Fig. $2 \mathrm{~A}, \mathrm{C}$, and D). No significant differences in neurolog-

A

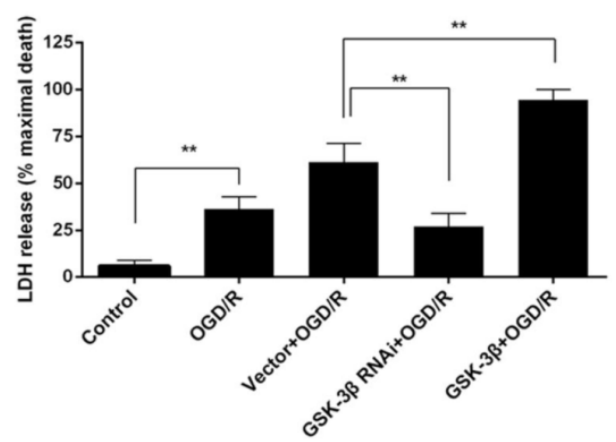

ical deficits and cerebral infarct volume were observed between the MCAO and scramble + MCAO groups. Brain water content was $78.40 \pm 0.77 \%$ in the sham group (Fig. 2B). In the GSK-3 $\beta$ RNAi + MCAO group, brain water content was $79.02 \pm 0.62 \%$, which was lower than in the MCAO $(82.92 \% \pm 0.62 \%)$ and scramble + MCAO groups $(82.48 \pm 0.38 \%)$. There was no significant difference in brain water content between the MCAO and scramble + MCAO groups.

Figure 1. Effects of GSK-3 $\beta$ on injury and survival of cortical neurons subjected to oxygen-glucose deprivation/reoxygenation (OGD/R). Neurons were transfected with GSK-3 $\beta$ siRNA and overexpression virus $72 \mathrm{~h}$ before OGD/R. A, Cell viability was measured by MTS assay. Data were normalized by defining control as $100 \%$

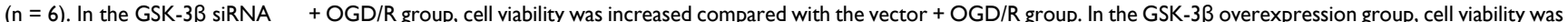
decreased compared with the vector + OGD/R group. B, Cell injury was detected by LDH assay $(n=6)$. The release of LDH was decreased in the GSK-3 $\beta$ siRNA + OGD/R group compared with the vector + OGD/R group. In the GSK-3 $\beta$ overexpression group, LDH release was significantly increased compared with the vector + OGD/R group. *P $<0.05, * * P<0.01$.

A

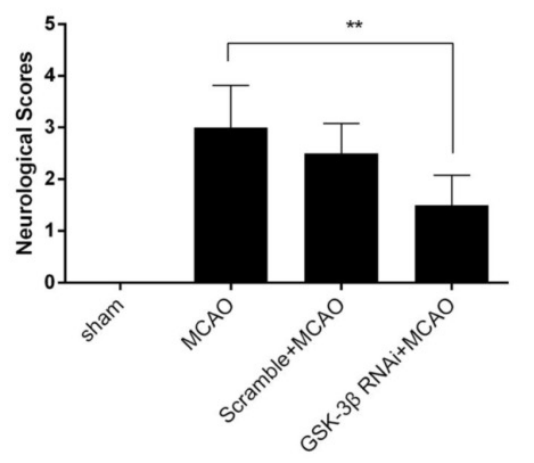

C

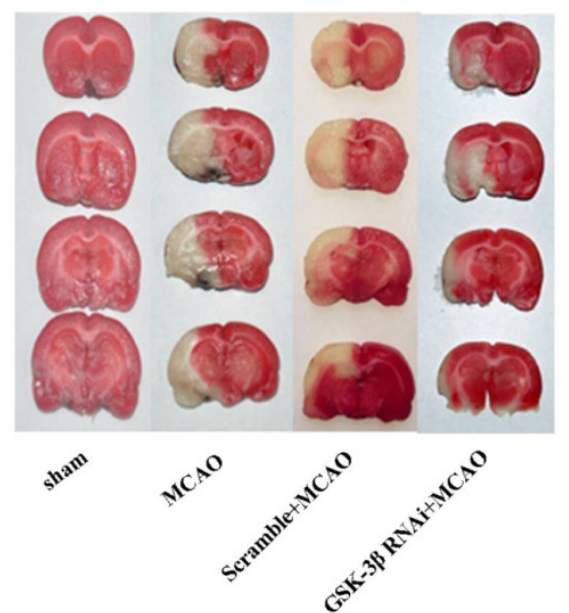

B

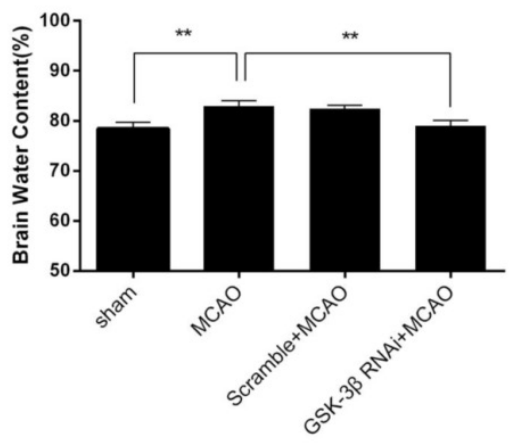

D

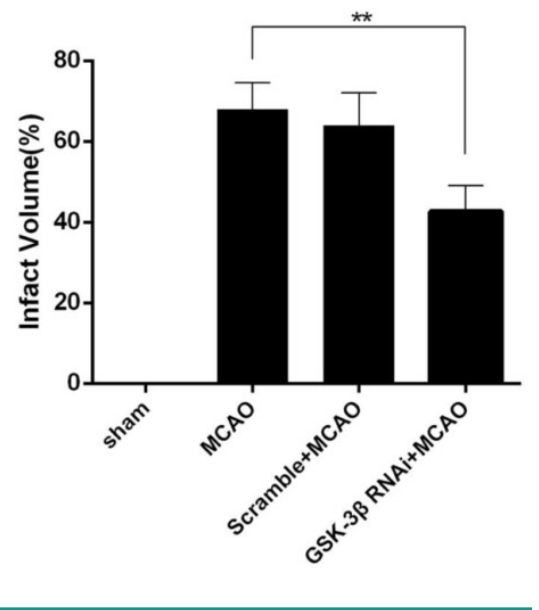




\section{Effects of GSK-3 $\beta$ on Morphological Changes of Cerebral Neurons in Rats}

Morphological changes of cerebral neurons were evaluated by HE and Nissl-staining. The number of intact neurons was counted and neuronal phenotypes were evaluated in the penumbra. HE staining demonstrated that there were more intact neurons in the GSK-3 $\beta$ interference group than in the MCAO group (Fig. 3A-D). In addition, fewer atrophic neurons that showed damaged nuclei and less shrunken cytoplasm were observed in the GSK- $3 \beta$ interference group. No significant differences were observed between the MCAO and scramble + MCAO groups. The results from Nissl staining were similar to those from HE staining. More Nissl bodies were observed in the GSK-3 $\beta$ interference group than in the MCAO and scramble $+\mathrm{MCAO}$ groups. Thus, the number of intact neurons was higher in the GSK-3 $\beta$ interference group compared with the MCAO group (Fig. 4A-E).
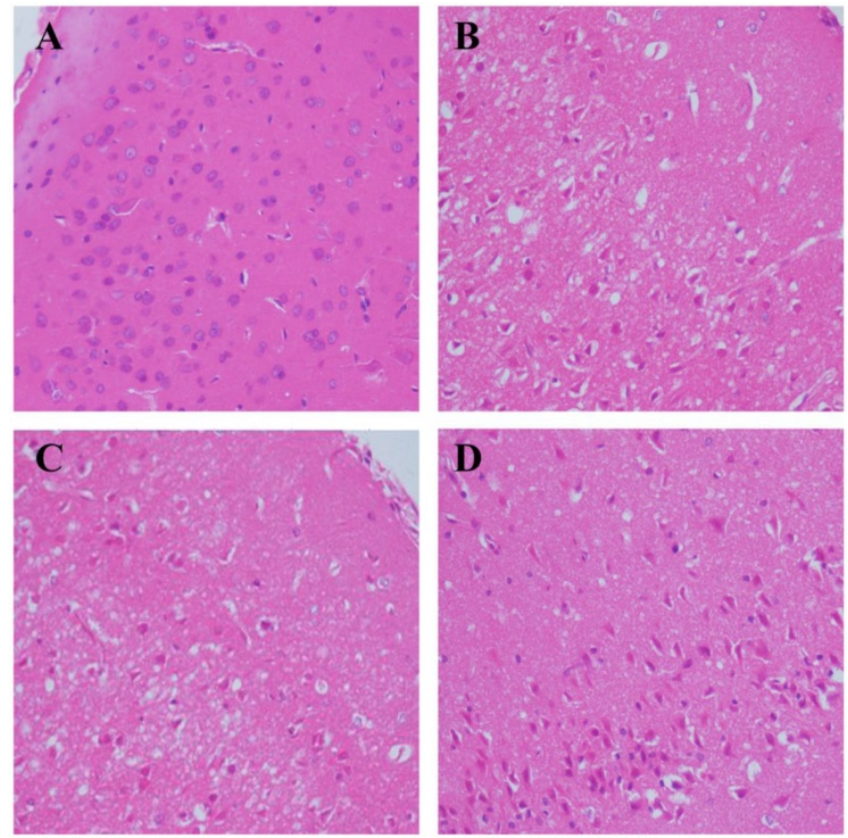

Figure 3. Effects of GSK-3 $\beta$ on morphological changes of cerebral cortical neurons. Hematoxylin-eosin (HE) staining was used to observe morphological changes of cerebral neurons ( $n=6$ per group). Magnification $\times 400$. A, Sham group. Brain tissue was dense and nuclei were intact and clear. $B$ and $C, M C A O$ and scramble

+ MCAO groups. Brain tissue was loose and many cells in the penumbra showed nuclear changes. D, GSK-3 $\beta+$ MCAO group. Brain tissue was denser and the number of intact neurons was increased compared with the $M C A O$ group. $M C A O=$ middle cerebral artery occlusion.

\section{Effects of GSK-3 $\beta$ on Expression of Nrf2 in Neurons}

In the GSK-3 $\beta$ siRNA + OGD/R group, total neuronal Nrf2 (Fig. 5C) as well as neuronal intranuclear Nrf2 (Fig. 5D) increased compared with the vector + OGD $/ \mathrm{R}$ and $\mathrm{OGD} / \mathrm{R}$ groups. The changes were similar to the curcumin + OGD/R group. We then used leptomycin $\mathrm{B}$, a nuclear transfer inhibitor, to detect changes in Nrf2. Total Nrf2 (Fig. 6A) and intranuclear Nrf2 (Fig. 6B) decreased in the GSK-3 $\beta$ overexpression group compared with the vector + OGD/R and OGD/R groups. However, in the GSK-3 $\beta$ overexpression + leptomycin B + OGD/R group, GSK-3 $\beta$ increased compared with the GSK-3 $\beta$ overexpression group. In addition, no significant changes in total Nrf2 or intranuclear Nrf2 were observed in all groups in the absence of OGD/R (Fig 5A, B).
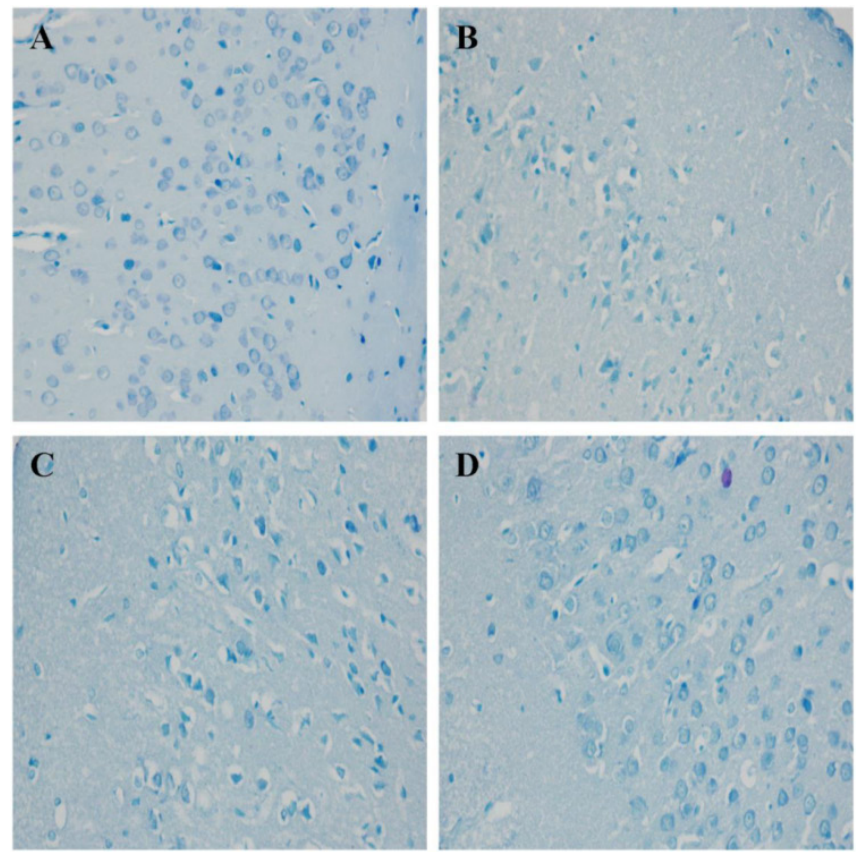

E

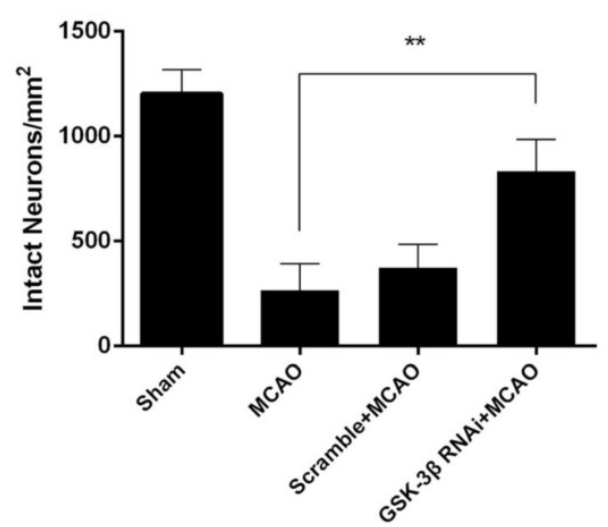

Figure 4. Nissl staining was conducted $24 \mathrm{~h}$ after focal cerebral ischemia in rats ( $n=6$ per group). Magnification $\times 400$. A, In the sham group, neuronal nuclei were intact and Nissl bodies in the cytoplasm were obvious. B and C, In the MCAO and scramble + MCAO groups, most neurons in the penumbra showed shrinkage and nuclear pyknosis. Most neurons and Nissl bodies disappeared and the intercellular space was enlarged. D, In the GSK-3 $\beta$ siRNA group, the number of intact neurons increased and Nissl bodies were obvious compared with the MCAO and scramble + $M C A O$ groups. $E$, Intact neurons were counted to measure survivability. The number of intact neurons in the GSK-3 $\beta$ group was significantly higher than in the MCAO and scramble + MCAO groups $(* * \mathrm{P}<0.01, \mathrm{n}=6)$. MCAO $=$ middle cerebral artery occlusion. 

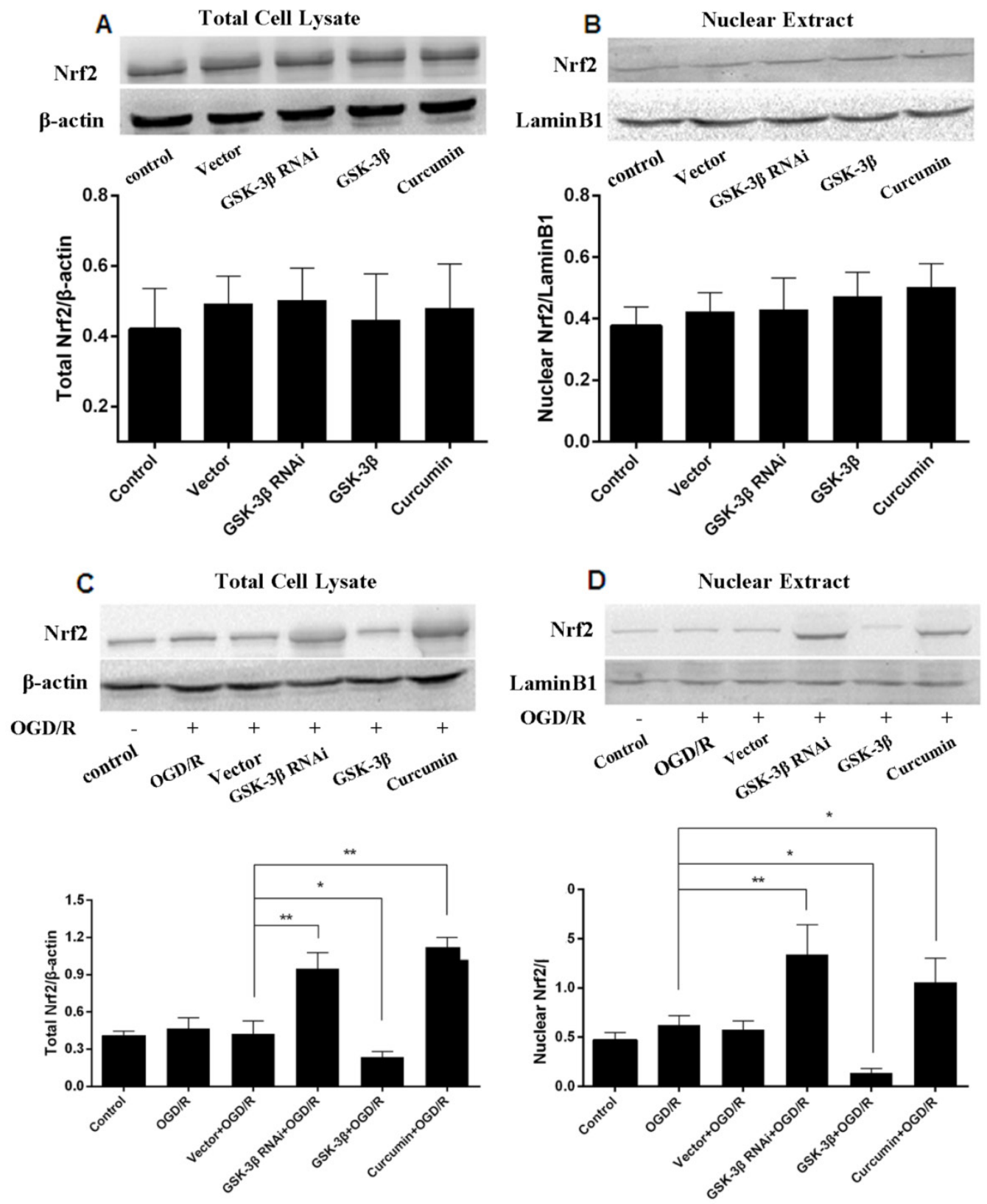

Figure 5. Effects of GSK-3 $\beta$ on expression of Nrf2 in neurons analyzed by western blot. Total cell lysate and nuclear extracts were used to conduct western blot analysis of total Nrf2 and nuclear Nrf2, respectively. A and B, Neurons were assessed in the absence of oxygen-glucose deprivation/reoxygenation (OGD/R). No significant differences were observed between all groups. $C$ and D, In the GSK-3 3 siRNA + OGD/R group, total Nrf2 and nuclear Nrf2 were increased compared with the vector + OGD/R group. Total Nrf2 and nuclear Nrf2 decreased in the GSK-3 $\beta$ overexpression group compared with the vector + OGD/R group. Curcumin was used as a positive control. The bar graph illustrates the effects of GSK-3 $\beta$ siRNA or overexpression on expression of Nrf2 and nuclear Nrf2. Values are expressed as mean \pm SEM. $* \mathrm{P}<0.05 ; * * \mathrm{P}<0.01$. $\mathrm{n}=6$ per group.

An immunofluorescence assay was also used to detect total Nrf2 and nuclear Nrf2 in neurons. As shown in Fig. 7A-E, expression of Nrf2 and nuclear Nrf2 was higher in the GSK-3 $\beta$ siRNA + OGD/R group compared with the OGD/R group. In contrast, total Nrf2 and nuclear Nrf2 decreased significantly in the GSK-3 $\beta$ overexpression + OGD/R group, compared with the OGD/R group. These results suggest that GSK-3 $\beta$ participated in the distribution of Nrf2 in the nucleus when neurons were subjected to OGD/R.

\section{Effects of GSK-3 $\beta$ on Expression of Nrf2 in the Cerebral Cortex of Rats}

Western blots were used to quantitate total Nrf2 in cerebral cortical cells and nuclei. Similar to the curcumin + MCAO group, total Nrf2 and intranuclear Nrf2 increased in the GSK-3 $\beta$ interference group, compared with the MCAO and scramble + MCAO groups (Fig. 8A, B). No significant differences were detected between the MCAO and scramble groups. In addition, no significant differences in total Nrf2 or 
intranuclear Nrf2 in the cerebral cortex were observed in all groups in the absence of MCAO (Fig. 8C, D).

Total Nrf2 and nuclear Nrf2 in rat cerebral cortical neurons were also examined by immunofluorescence assay. As shown in Fig. 9A-D, expression of Nrf2 in the GSK-3 $\beta+$ MCAO group increased significantly compared with the MCAO and scramble + MCAO groups. No significant difference was observed between the MCAO and scramble + MCAO groups.

\section{Effects of GSK-3 $\beta$ on Expression of Srxl and Trx 1}

The phase II detoxifying enzymes and antioxidants Srx1 and Trx1 were detected in neurons after GSK-3 $\beta$ interference or overexpression before OGD/R (Fig. 10A). Srx1 and Trx1 levels increased in the GSK-3 $\beta$ interference group compared with the OGD/R and vector virus groups. GSK-3 $\beta$ overexpression decreased expression of Srx1 and Trx1 in neurons. GSK-3 $\beta$ interference increased levels of Srx1 and Trx 1 in the rat cerebral cortex compared with the MCAO and scramble + MCAO groups (Fig. 10B).

\section{Effects of GSK-3 $\beta$ on SOD and MDA}

The effects of GSK-3 $\beta$ siRNA on neuronal SOD and MDA levels are shown in Fig. 11A and B. A significant decrease in neuronal SOD activity was detected in the OGD/R group (Fig. 11A). SOD activity in the GSK-3 $\beta$ siRNA + OGD/R group was higher compared with the vector $+\mathrm{OGD} / \mathrm{R}$ group. In the GSK-3 $\beta$ overexpression + OGD/R group, SOD activity decreased compared with the vector + OGD/R group. MDA levels exhibited opposite changes (Fig. 11B). The effects of GSK- $3 \beta$ on the cerebral cortex SOD and MDA levels are shown in Fig. 11C and D. SOD activity decreased in the MCAO group compared with the sham group (Fig. 11C). Rats pretreated with GSK-3 $\beta$ siRNA before MCAO showed an increase in SOD activity compared with the MCAO group. Conversely, MDA levels in the cortex increased in the MCAO group compared with the control group, and decreased in the GSK-3 $\beta$ group compared with the MCAO group (Fig. 11D).
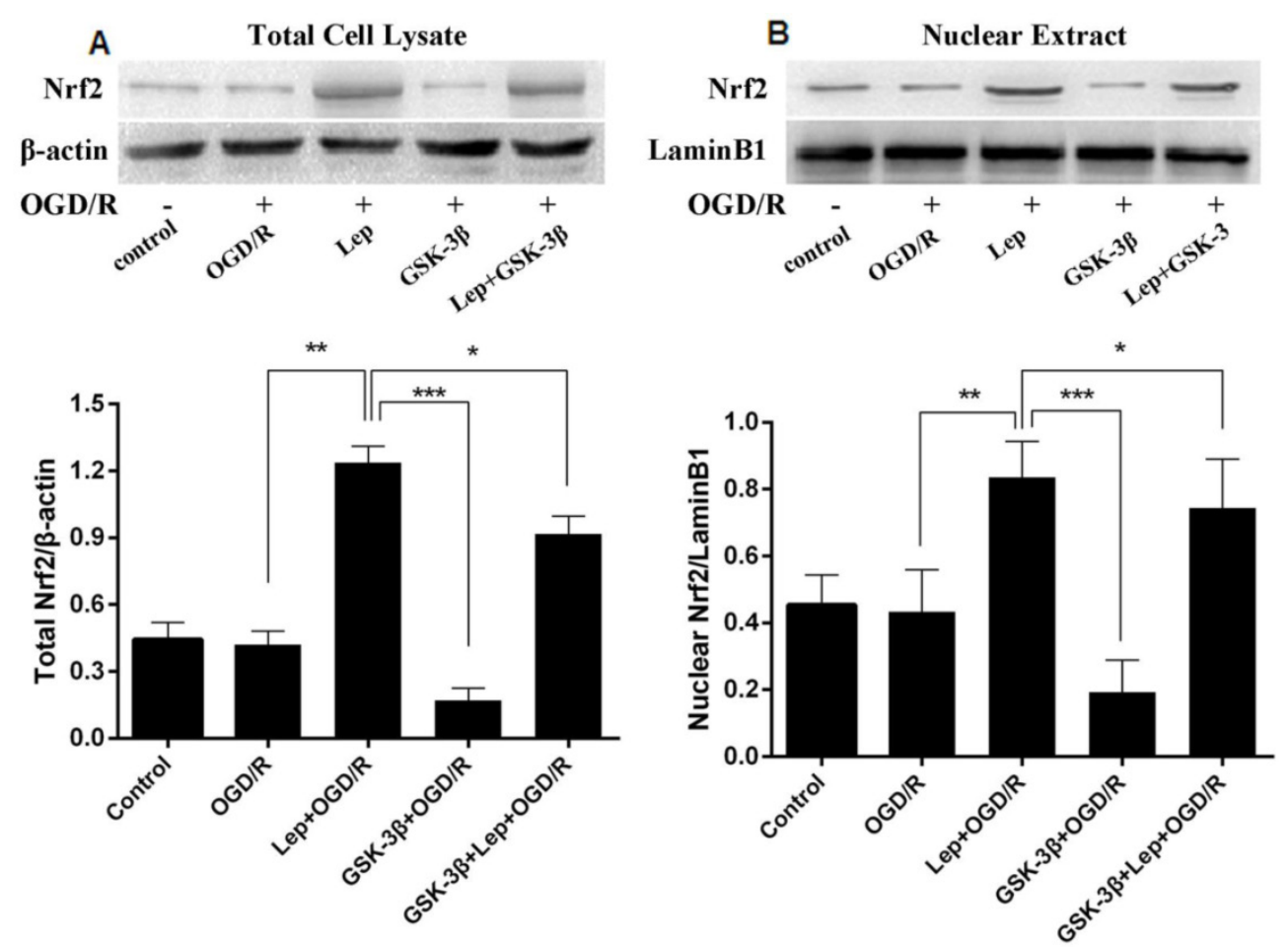

Figure 6. Effects of GSK-3 $\beta$ on expression of Nrf2 in neurons analyzed by western blot. A, In the leptomycin B + OGD/R group, total Nrf2 was increased compared with the OGD/R group. Total Nrf2 and nuclear Nrf2 decreased in the GSK-3 $\beta$ overexpression group but increased in the GSK-3 $\beta$ overexpression + leptomycin B + OGD/R group (GSK-3 $\beta$ + lep + OGD/R). B, Nuclear Nrf2 showed similar changes to total Nrf2 in all groups. The bar graph illustrates the effects of GSK-3 $\beta$ overexpression or leptomycin $\mathrm{B}$ on expression of nuclear Nrf2. Values are expressed as mean \pm SEM. $* \mathrm{P}<0.05 ; * * \mathrm{P}<0.01 ; * * * \mathrm{P}<0.001$. $\mathrm{n}=6$ per group. 


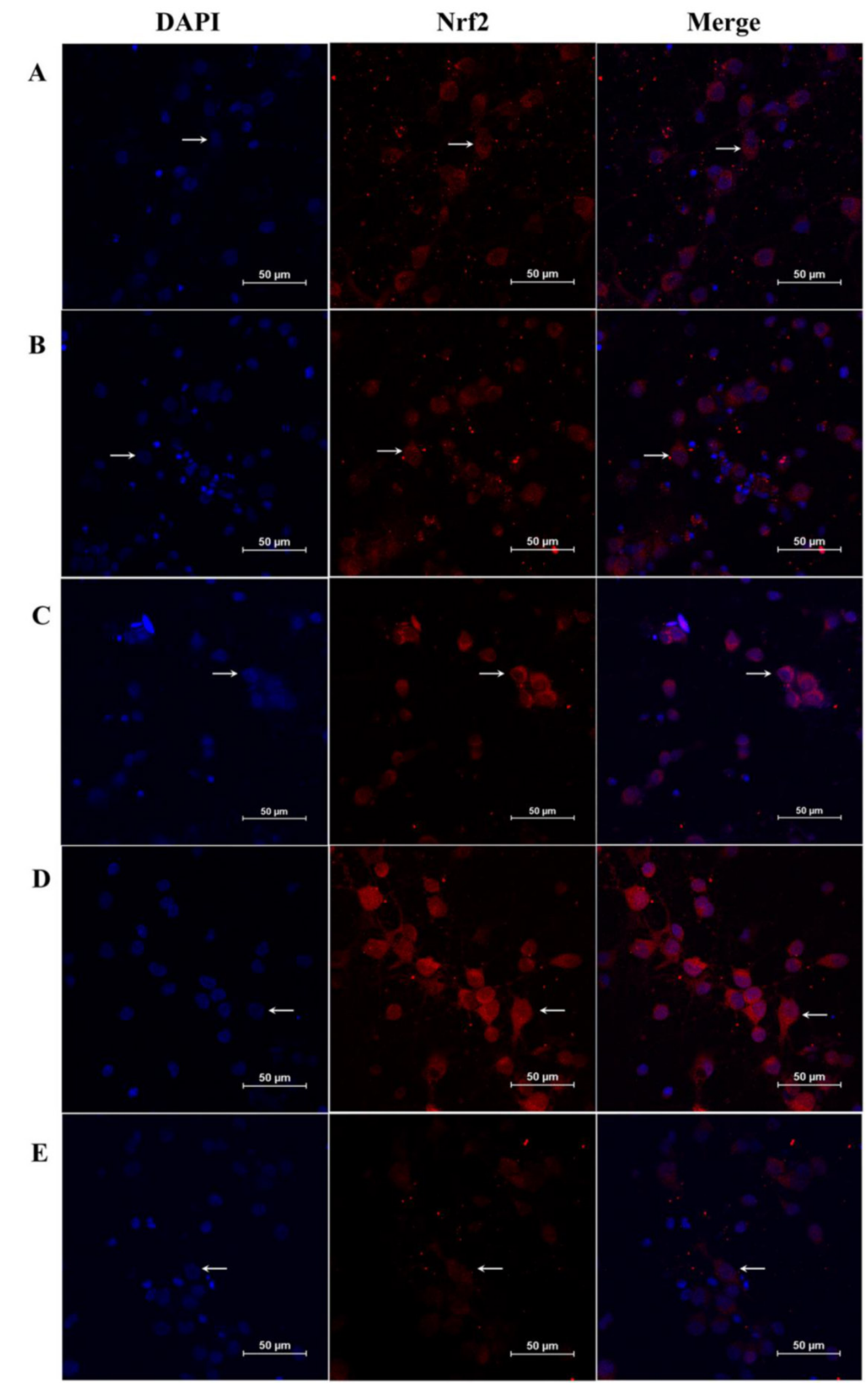

Figure 7. Effects of GSK-3 $\beta$ on expression of Nrf2 in neurons analyzed by immunofluorescence. Immunofluorescence assays were used to detect $\mathrm{Nrf2}$ in neurons. A laser scanning confocal microscope (LSCM) was used to detect fluorescent expression. A, Control group. B, OGD/R group. C, Vector + OGD/R group. D, GSK-3B siRNA + OGD/R group. E, GSK-3ß overexpression + OGD/R group. Nrf2 expression in neurons is indicated by red fluorescence. Neuronal nuclei were stained using DAPI. Original magnification, $\times 600$. The expression of Nrf2 was increased in the GSK-3 $\beta$ interference group and attenuated by GSK-3 $\beta$ overexpression. OGD/R $=$ oxygen-glucose deprivation/reoxygenation. 

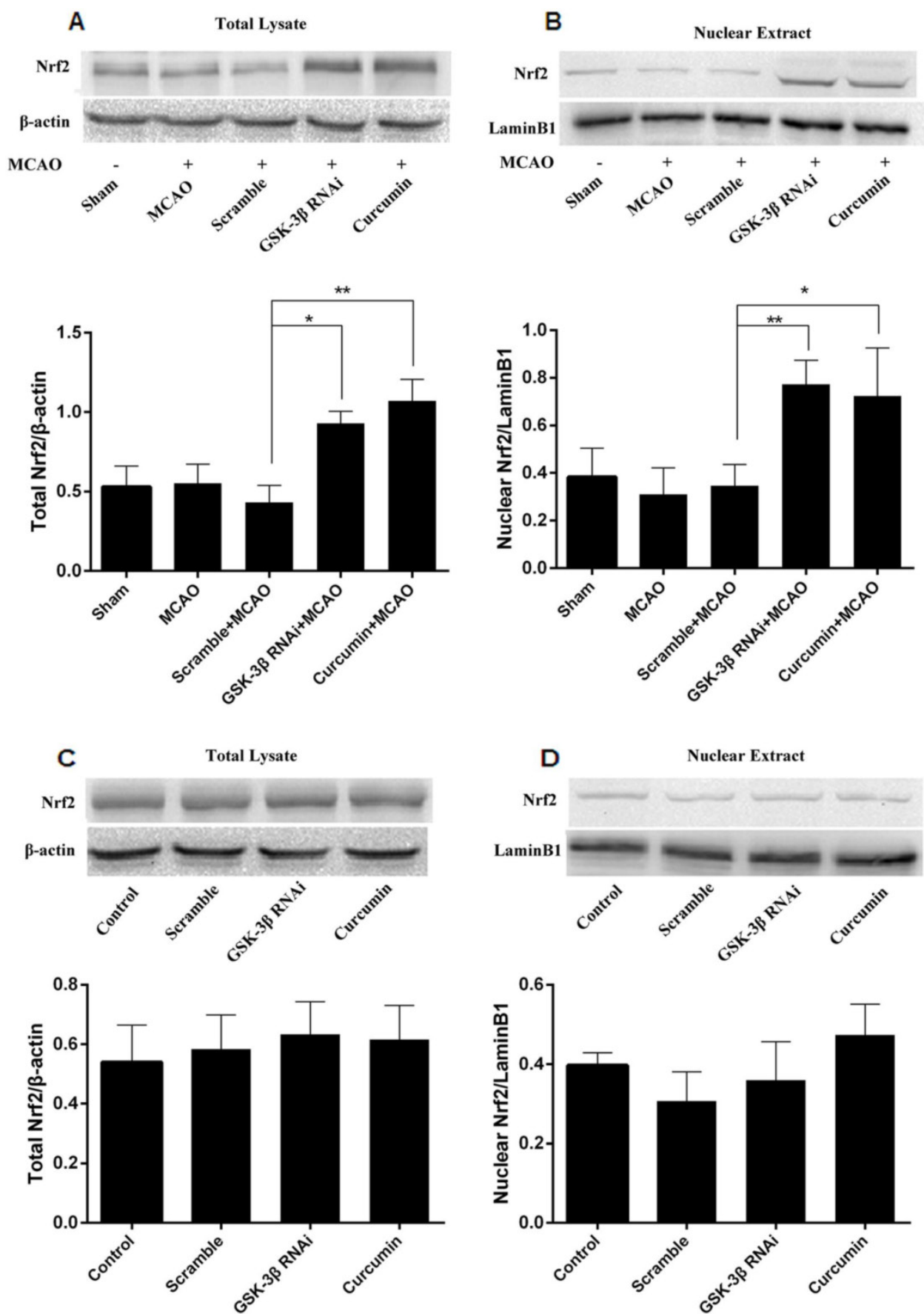

Figure 8. Effects of GSK-3 $\beta$ on expression of Nrf2 in the cerebral cortex of rats analyzed by western blot. Expression levels of total Nrf2 (A) and nuclear Nrf2 (B) in brain tissues from the injured cerebral hemisphere were detected by western blot. Total Nrf2 and nuclear Nrf2 were significantly higher in the GSK-3 $\beta$ interference group than in the MCAO and scramble + MCAO groups. The curcumin + MCAO group was used as positive control. No significant change in total Nrf2 and nuclear Nrf2 was detected in the absence of $\mathrm{MCAO}(\mathrm{C}$ and $\mathrm{D}) . \mathrm{n}=6$ per group, $* \mathrm{P}<0.05$, $* * \mathrm{P}<0.01$. MCAO = middle cerebral artery occlusion.

\section{Discussion}

Our study showed that inhibition of GSK-3 $\beta$ improves the survival rate of primary cortical neurons subjected to OGD/R and reduces OGD/R-induced cell injury in vitro. Moreover, our results also suggest that GSK-3 $\beta$ interference ameliorates neurological deficits, reduces brain infarct volume and water content, and reduces damage to cerebral cortical neurons after MCAO in rats. GSK-3 $\beta$ interference attenuates oxidative stress in neurons and rat cerebral cortex, whereas overexpression of GSK-3 $\beta$ exacerbates oxidative stress in neurons. Furthermore, we demonstrated that the neuroprotective effects of GSK-3 $\beta$ interference might be mediated by regulation of $\mathrm{Nrf} 2$.

GSK-3 $\beta$, which is a serine/threonine protein kinase, is a crucial regulator of neuronal fate, survival, or death in normal and pathological physiology [27]. Previous studies have shown that GSK- $3 \beta$ participates 
in the viability of various cells. Our study explored its effects on the viability and injury of neurons subjected to OGD/R. We suggest that knockdown of GSK-3 $\beta$ increases viability and improves injury induced by OGD/R, whereas overexpression of GSK-3 $\beta$ yields opposite results. A previous study also demonstrated that inhibition of GSK-3 $\beta$ alleviated acute hepatic damage induced by oxidative stress [3]. SOD and MDA, which are 2 important oxidative stress indicators, were used to investigate oxidative damage. Our results show that GSK-3 $\beta$ interference attenuates oxidative stress in vivo and in vitro. Moreover, research has demonstrated that administration of GSK-3 $\beta$ inhibitors decreased infarct size in the myocardium [8, 9]. Consistent with this result, our study showed that GSK-3 $\beta$ interference improved cerebral ischemia-reperfusion injury in rats. Overexpression of GSK-3 $\beta$ was not conducted in vivo due to low transfection efficiency.

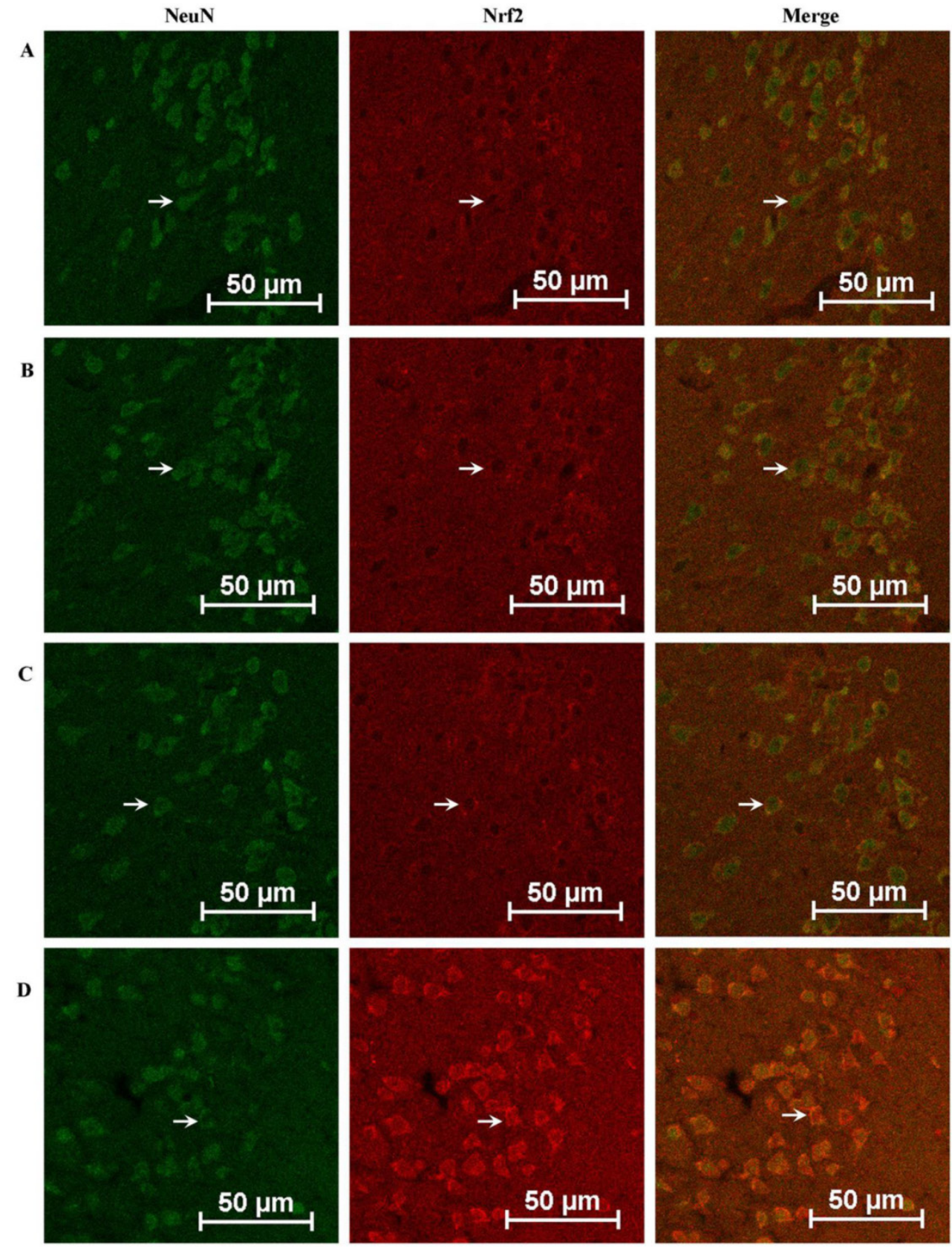

Figure 9. Effects of GSK-3 3 on expression of Nrf2 in the cerebral cortex of rats analyzed by immunofluorescence. The expression of Nrf2 in the cerebral cortex of rats was detected by immunofluorescence assay. A, Sham group. B, MCAO group. C, Scramble + MCAO group. D, GSK-3B interference group. Nrf2 expression is indicated by red fluorescence and cerebral cortical neurons are indicated by green fluorescence. The bar is $50 \mu \mathrm{m}$. The expression of Nrf2 in the GSK-3 $\beta$ interference group was higher than in any other group. MCAO = middle cerebral artery occlusion. 

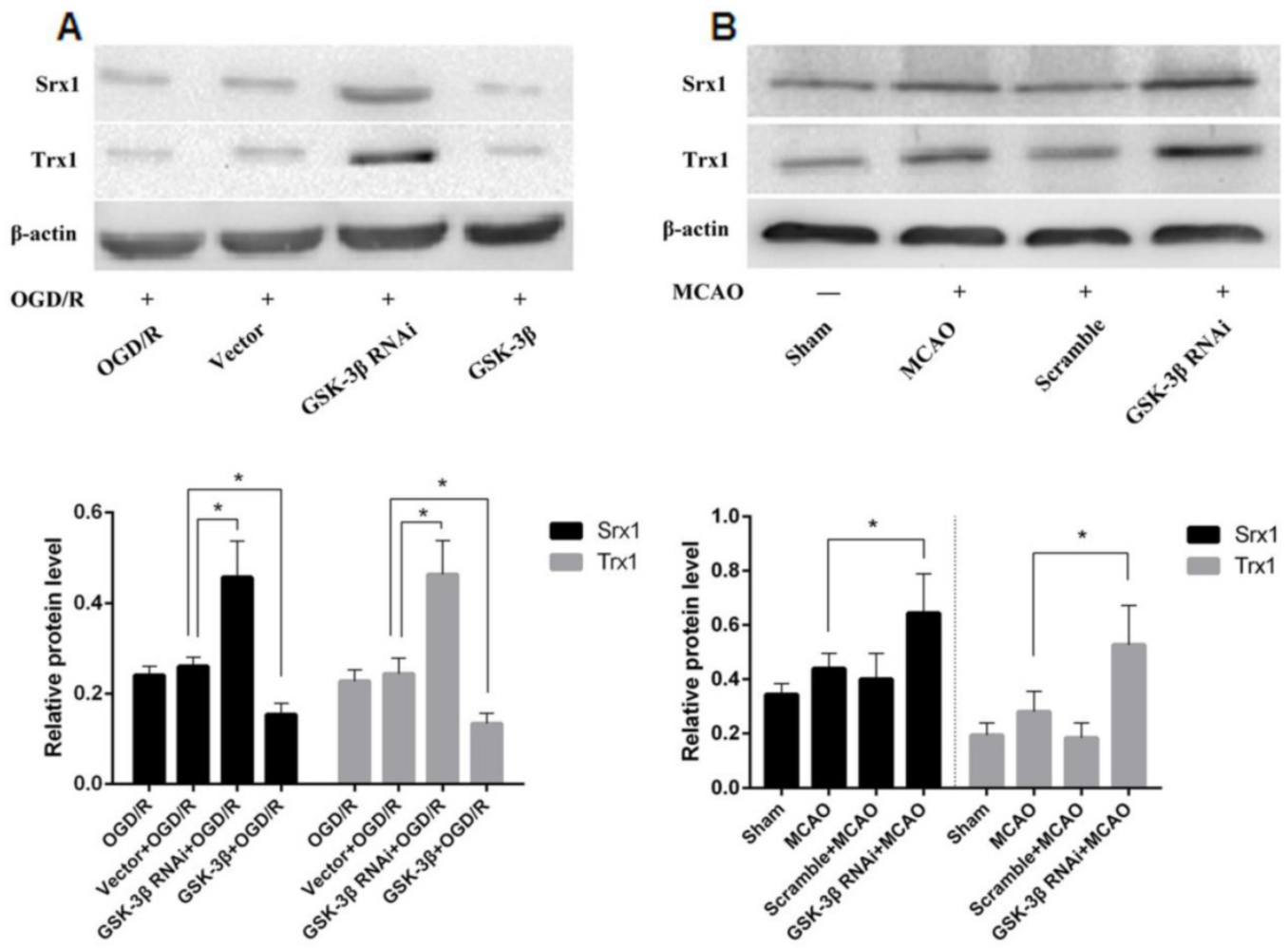

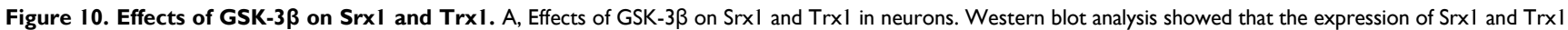
increased significantly in the GSK-3 $\beta$ interference group compared with the OGD/R and vector + OGD/R groups. The GSK-3 3 overexpression group presented opposite results. $B$, Effects of GSK-3 $\beta$ on Srxl and Trxl in cerebral tissue. Similar to the results in vitro, after the cerebrum was transfected with GSK-3 $\beta$ siRNA $48 \mathrm{~h}$ before MCAO, Srxl and Trxl increased compared with the MCAO and scramble + MCAO groups. Bar graphs show the relative protein levels of Srxl and Trx 1 standardized with $\beta$-actin. $n=6$ per group; $* P$ $<0.05$; **P $<0.01$. OGD/R = oxygen-glucose deprivation/reoxygenation, MCAO = middle cerebral artery occlusion.
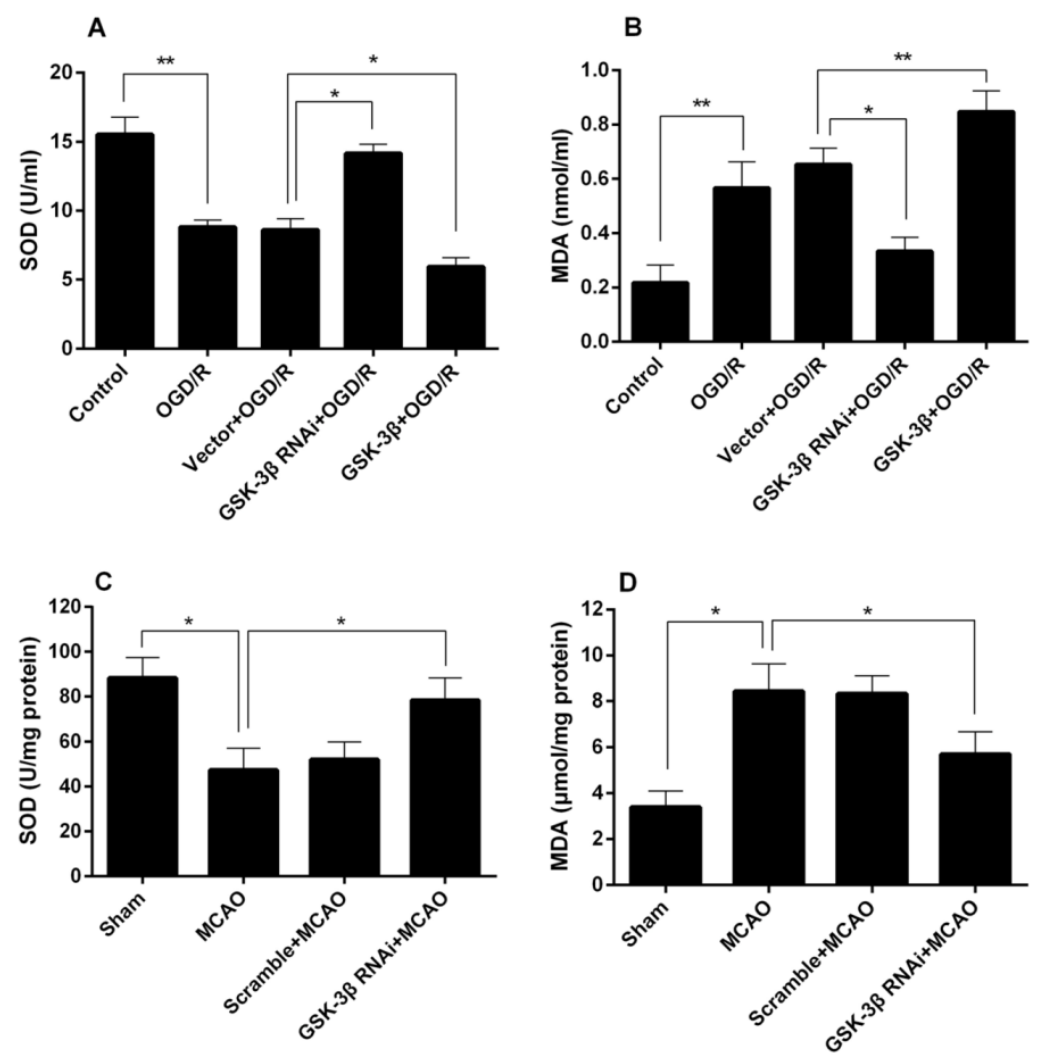

Figure 11. Effects of GSK-3 $\beta$ on SOD and MDA. A and B, Effects of GSK-3 $\beta$ on neuronal SOD and MDA levels. C and D, Effects of GSK-3 $\beta$ on rat cortex SOD and MDA levels. $\mathrm{n}=6$ per group; $* \mathrm{P}<0.05 ; * * \mathrm{P}<0.01$. OGD/R = oxygen-glucose deprivation/reoxygenation, $\mathrm{MCAO}=$ middle cerebral artery occlusion. 
As shown in previous studies, GSK-3 $\beta$ participates in subcellular translocation of Nrf2 to the nucleus. Nrf2, which is a key factor in the oxidative stress response [11], regulates expression of phase II detoxifying enzymes and antioxidants, such as Srx1 and Trx1, through the Nrf2/ARE pathway. These inductions are significant for the protection of cells against oxidative stress [14]. Nrf2 is critical for the protection of neurons from oxidative injury induced by ischemia/reperfusion as described in our previous study [16]. Current studies indicate that GSK-3 $\beta$ participates in regulation of Nrf2. Therefore, we hypothesize that the neuroprotective effects of GSK-3 $\beta$ interference are related to the expression and subcellular localization of Nrf2. In this study, we demonstrated that knockdown of GSK-3 $\beta$ in vivo and in vitro increased expression of Nrf2 and nuclear Nrf2. Overexpression of GSK-3 $\beta$ yielded opposite results in neurons. Curcumin induces Nrf2 expression [28] and curcumin treatment of neurons and rats exerted effects similar to GSK-3 $\beta$ interference.

Leptomycin B is a nuclear export inhibitor that blocks nuclear export of Nrf2 in liver cancer cells [29, 30]. Our study showed that total Nrf2 and nuclear Nrf2 increased after neurons were treated with leptomycin B. Nrf2 is retained and degraded mainly in the cytoplasm by Keap1 [31, 32]. Thus, we hypothesize that the increase of total Nrf2 was due to accumulation of Nrf2 in the nucleus induced by leptomycin B and decreased degradation of Nrf2 in the cytoplasm. However, total Nrf2 and nuclear Nrf2 levels decreased after combined GSK-3 $\beta$ overexpression and leptomycin $\mathrm{B}$ treatment before $\mathrm{OGD} / \mathrm{R}$ in neurons, compared with leptomycin B treatment alone. In addition, levels of Nrf2 downstream proteins Srx1 and Trx1 increased after GSK-3 $\beta$ interference, whereas overexpression of GSK-3 $\beta$ downregulated expression of Srx1 and Trx1 in vitro. Similarly, knockdown of GSK-3 $\beta$ in vivo increased expression of Srx1 and Trx1. Leptomycin B was not used in vivo due to unknown effects. Thus, GSK- $3 \beta$ may participate in the distribution of Nrf2 inside and outside of the nucleus. A previous study indicated that GSK-3 $\beta$ regulates nuclear export and degradation of Nrf2 through Fyn kinase in human hepatoma (HepG2) cells [17]. Moreover, SCF/-TrCP promotes GSK-3 $\beta$ degradation of Nrf2 in human embryonic kidney (HEK) 293T cells [18]. However, the specific mechanisms through which GSK-3 $\beta$ regulates Nrf2 in cerebral ische$\mathrm{mia} /$ reperfusion have not been clarified and require further study.

In summary, our study suggests that GSK-3 $\beta$ influences injury due to cerebral ischemia/reperfusion. These effects may be mediated by its negative regula- tion of Nrf2. Our results indicate a potential new therapeutic target for clinical treatment of stroke.

\section{Abbreviations}

ANOVA: analysis of variance; ARE: antioxidant responsive element; MCAO: middle cerebral artery occlusion; Nrf2: nuclear factor erythroid 2-related factor 2; OGD/R: oxygen-glucose deprivation/reoxygenation; Srx1: sulfiredoxin; Trx-1: thioredoxin-1; HepG2: human hepatoma; HEK293T: human embryo kidney 293T; LDH: Lactate dehydrogenase; MTS: colorimetric 3-(4,5-dimethylthiazol-2yl)-5(3-carboxymethoxyphenyl)-(4-sulfophenyl)-2H-tetraz olium; TTC: 2,3,5-triphenyl tetrazolium chloride; SOD: superoxide dismutase; MDA: malondialdehvde.

\section{Acknowledgments}

This work was supported by The Natural Science Youth Foundation of China (No.81301125), Natural Science Foundation of China (No. 81271460), and Natural Science Foundation of Chongqing Education Committee, China (No. KJ1500230).

\section{Competing Interests}

No conflict of interest exits in the submission of this manuscript, and all the authors listed have approved the manuscript.

\section{References}

1. Donnan GA, Fisher M, Macleod M, et al. Stroke. Lancet. 2008; 371: 1612-1623.

2. Liesz A, Sun L, Zhou W, et al. FTY720 reduces post-ischemic brain lymphocyte influx but does not improve outcome in permanent murine cerebral ischemia. PLoS One. 2011; 6: e21312.

3. Linlin Wei, Feng Ren, Xiangying Zhang, et al. Oxidative stress promotes D-GalN/LPS-induced acute hepatotoxicity by increasing glycogen synthase kinase 3b activity. Inflamm Res. 2014; 63: 485-494

4. Woodgett J. R. Recent advances in the protein kinase B signaling pathway. Curr. Opin. Cell Biol. 2005; 17: 150-157.

5. Haiyan Tong, Kenichi Imahashi, Charles Steenbergen, et al. Phosphorylation of glycogen synthase kinase-3beta during preconditioning through a phosphatidylinositol-3-kinase-dependent pathway is cardioprotective. Circ Res. 2002; 90: 377-379.

6. Nishihara M, Miura T, Miki T, et al. Erythropoietin affords additional cardioprotection to preconditioned hearts by enhanced phosphorylation of glycogen synthase kinase-3 beta. Am J Physiol Heart Circ Physiol. 2006; 291: H748-H755

7. Gomez L, Paillard M, Thibault H, et al. Inhibition of GSK3beta by postconditioning is required to prevent opening of the mitochondrial permeability transition pore during reperfusion. Circulation. 2008; 117: 2761-2768.

8. Gross ER, Hsu AK, Gross GJ. Opioid-induced cardioprotection occurs via glycogen synthase kinase beta inhibition during reperfusion in intact rat hearts. Circ Res. 2004; 94: 960-966.

9. Park SS, Zhao H, Mueller RA, et al. Bradykinin prevents reperfusion injury by targeting mitochondrial permeability transition pore through glycogen synthase kinase 3beta. J Mol Cell Cardiol. 2006; 40: 708-716.

10. Salazar, M., A. I. Rojo, D. Velasco, R. M. et al. Glycogen synthase kinase-3beta inhibits the xenobiotic and antioxidant cell response by direct phosphorylation and nuclear exclusion of the transcription factor Nrf2. J. Biol. Chem. 2006; 281: 14841-14851.

11. Kaspar JW, Niture SK, Jaiswal AK. Nrf2:INrf2(Keap1) Signaling in Oxidative Stress. Free radical biology \& medicine. 2009; 47(9): 1304-1309.

12. Soriano FX, Léveillé F, Papadia S, et al. Induction of sulfiredoxin expression and reduction of peroxiredoxin hyperoxidation by the neuroprotective Nrf2 activator 3H-1, 2-dithiole-3-thione. J Neurochem. 2008; 107: 533-543.

13. Tanito M, Agbaga MP, Anderson RE. Upregulation of thioredoxin system via Nrf2-antioxidant responsive element pathway in adaptive-retinal neuroprotection in vivo and in vitro. Free Radic Biol Med. 2007; 42: 1838-1850.

14. Jaiswal, A. K. Nrf2 signaling in coordinated activation of antioxidant gene expression.Free Radic. Biol. Med. 2004; 36: 1199-1207. 
15. Bo Chen, Yanrong $\mathrm{Lu}$, Younan Chen, et al. The role of Nrf2 in oxidative stress-induced endothelial injuries. J Endocrinol. 2015;225: R83-R99.

16. Wu J, Li Q, Wang $X$, et al. Neuroprotection by Curcumin in Ischemic Brain Injury Involves the Akt/Nrf2 Pathway. PLoS ONE. 2013; 8(3): e59843.

17. Abhinav K. Jain and Anil K. Jaiswal. GSK-3 Acts Upstream of Fyn Kinase in Regulation of Nuclear Export and Degradation of NF-E2 Related Factor 2. J. Biol. Chem. 2007; 282: 16502-16510.

18. Rada P, Rojo AI, Chowdhry S, et al. SCF/-TrCP Promotes Glycogen Synthase Kinase 3-Dependent Degradation of the Nrf2 Transcription Factor in a Keap1-Independent Manner. Molecular and Cellular Biology. 2011; 31(6): 1121-33

19. Rojo, A. I., M. R. Sagarra, and A. Cuadrado. GSK-3beta down-regulates the transcription factor Nrf2 after oxidant damage: relevance to exposure of neuronal cells to oxidative stress. J. Neurochem. 2008; 105: 192-202.

20. Ming $\mathrm{Y}$, Zhang H, Long L, et al. Modulation of Ca2+ signals by phosphatidylinositol-linked novel D1 dopamine receptor in hippocampal neurons. J Neurochem. 2006; 98: 1316-1323.

21. $\mathrm{Wu} \mathrm{X}, \mathrm{Zhao} J, \mathrm{Yu} \mathrm{S}$, et al. Sulforaphane protects primary cultures of cortical neurons against injury induced by oxygen-glucose deprivation/reoxygenation via antiapoptosis. Neurosci Bull. 2012; 28: 509-516.

22. Yu SS, Zhao J, Zheng WP, et al. Neuroprotective effect of 4-hydroxybenzyl alcohol against transient focal cerebral ischemia via anti-apoptosis in rats. Brain Res. 2010; 1308: 167-175.

23. Chen Y, Wu X, Yu S, et al. Neuroprotection of Tanshinone IIA against Cerebral Ischemia/Reperfusion Injury through Inhibition of Macrophage Migration Inhibitory Factor in Rats. PLoS One. 2012; 7: e40165.

24. L. Li, K. Zhu, Y. Liu, et al. Targeting thioredoxin-1 with siRNA exacerbates oxidative stress injury after cerebral ischemia/reperfusion in rats. Neuroscience. 2015 ; 284: 815-823.

25. Longa EZ, Weinstein PR, Carlson S, et al. Reversibal middle cerebral artery occlusion without craniectomy in tats. Stroke. 1989; 20: 84-91.

26. Shanshan $\mathrm{Yu}$, Jing Zhao, Shipeng Lei, et al. 4-Hydroxybenzyl Alcohol Ameliorates Cerebral Injury in Rats by Antioxidant Action. Neurochem Res. 2011; 36: 339-346.

27. Jope RS, Johnson GV. The glamour and gloom of glycogen synthase kinase-3. Trends Biochem Sci Feb. 2004; 29(2): 95-102.

28. Wu JX, Zhang LY, Chen YL, et al. Curcumin pretreatment and post-treatment both improve the antioxidative ability of neurons with oxygen-glucose deprivation. Neural Regen Res. 2015; 10(3): 481-9.

29. Wtai $Y$, Kobayashi A, Nagase H, et al. Subcellular localization and cytoplasmic complex status of endogenous Keap1. Genes cell. 2007; 12(10):1163-78.

30. Sankaranarayanan Kannan, and Anil K. Jaiswal. Low and high dose UVB regulation of transcription factor NF-E2-related factor 2. Cancer Res. 2006; 66(17): 8421-9.

31. Itoh, K., Wakabayashi, N., Katoh, Y., et al. Keap1 represses nuclear activation of antioxidant responsive elements by Nrf2 through binding to the amino-terminal Neh2 domain. Genes Dev. 1999; 13: 76-86.

32. Dhakshinamoorthy, $\mathrm{S}$, and Jaiswal, AK. Functional characterization and role of INrf2 in antioxidant response element-mediated expression and antioxidant induction of NAD(P)H:quinone oxidoreductase1. Gene. 2001; 20(29): 3906-17. 